\title{
The Response Measures of Song Government to "Zhang-epidemic" in Bashu Area
}

\author{
Qian Wu \\ College of Historical Culture \& Tourism, Southwest Minzu University, Chengdu, Sichuan, China \\ Email: 337808290@qq.com
}

\begin{abstract}
During the Song Dynasty, "Zhang-epidemic" often occurred because of social activities, natural environment and other factors. As a high incidence area of "Zhang-epidemic" in Bashu area, the Song government adopted flexible and effective epidemic prevention measures according to its characteristics. This paper induction the epidemic situation of "Zhang-epidemic" in Bashu area in Song Dynasty, and analyzes the positive control measures taken by the government of Song Dynasty to control and deal with the epidemic situation. All of these provided the experience of prevention and control of "Zhang-epidemic" for the later dynasties and laid a foundation for the development of medical and health services in China to a certain extent.
\end{abstract}

Keywords: the two Song Dynasty, Bashu, "Zhang-epidemic", countermeasures

\section{Introduction}

"Zhang-epidemic", as a kind of local disease, shrouded the Bashu people who lived in the Song Dynasty. "Zhang-epidemic" epidemic rampant, to the social production and life, historical development process and natural ecological environment caused very serious damage, has a profound impact. Therefore, the central government of Song Dynasty actively took various measures to control, deal with and prevent the "Zhang-epidemic" epidemic, which occurred frequently in the southern, southwest and western border areas.

\section{Basic Knowledge of "Zhang-epidemic"}

From the perspective of history, scholars in the academic circle have made many achievements in the definition of "Zhang"; the distribution and change of "Zhang-epidemic"; the prevention and control measures of "Zhang-epidemic", and the influence of "Zhang-epidemic". Based on the needs of this study, the author will make a brief introduction from three aspects: the definition of "miasma", the distribution and change of "Zhang-epidemic" in Bashu area, and the impact of "Zhang-epidemic" on Bashu area.

First, the definition of "Zhang". As for the origin, definition and relationship between the names of "Zhang" and "Zhang-gas", ancient literature and modern scholars have different understanding and research. According to most opinions, "Zhang" refers to the pathogenic or toxic air with dense vegetation, hot and humid in the south, as well as the diseases caused by the gas. The concept of "Zhang" is rather complicated. Many scholars think that "Zhang" is a kind of miasma, especially malignant "Zhang-epidemic". There are many articles about the concept of "Zhang". For example, Peng Zuo's "discussion on the name and reality of "Zhang" " [1] proposed that the concept of "Zhang" appeared earlier than the word "Zhang". At first, "Zhang" was essentially a "barrier", and later "Zhang" was differentiated, which indicated the damp heat and steaming Qi which was prevalent in the southern region and made people sick. "Zhang" refers to miasma, especially falciparum miasma. Hong Zheng pointed out that "Zhang" has two meanings in the article "the change of "Zhang" concept and its influence on the life of ancient Lingnan" [2]. One is miasma disease, which is a generalization of various diseases in the natural geographical and climatic conditions of the south. The second is the pathogenic "Zhang-gas". In the concept of traditional Chinese medicine, "Zhang" originates from "barrier". Its etiological significance refers to the natural environmental factors that affect human health due to the blocked Qi mechanism. It is the characteristics of etiological theory of traditional Chinese medicine to summarize the influence of environmental and climatic factors on human body into some "Qi". In Yongmin Cai and Mengting Wang's 
textual "research and specification of "Zhang" name" [3], it is pointed out that "Zhang-gas" in ancient medical books has different concepts due to different contexts, sometimes referring to disease syndromes, sometimes referring to etiology. The modern "Zhang-gas" is all the evils that lead to human diseases, including miasma and beriberi. In Hanyong Feng's "literature study on "Zhang-gas"” [4], we classified the literature records on miasma, and found that "Zhang-epidemic" contains about ten kinds of diseases, such as miasma, dysentery, febrile disease, beriberi, sand damp heat, poisoning, laryngology, carbuncle and so on. Among them, the author thinks that "Zhang-gas" is a general term for a variety of diseases, so the names of "Zhang-gas" are also different, and there are as many as 40 that can be tested. Secondly, the research on the distribution and change of "Zhang-gas" in Bashu area. The special natural environment and geographical conditions in South China are the main causes of "Zhang-epidemic". According to "the preliminary study on the distribution and change of "Zhang-epidemic" in China in 2000" [5], the occurrence of "Zhang-epidemic" is related to geographical environment and climate. More because of human activities, the "Zhang-epidemic" epidemic area gradually moved south, and the "Zhang-epidemic" also changed from large to small, from heavy to light. According to the literature, the "Zhang-epidemic" in Song Dynasty mainly existed in Guangdong, Guangxi, Fujian, Jiangxi, Sichuan, Chongqing, Hunan, Hainan and other places. Jiawei Fan's article "population migration in the Six Dynasties and 'Zhang-epidemic' in Lingnan area" [6] analyzes the threat of local diseases faced by people who moved south and moved to Lingnan area in the Six Dynasties from the perspective of local disease infection and population migration. Third, the impact of "Zhang-epidemic" on Bashu area. In Peng Zuo's "'Zhang-epidemic' and cultural changes in the song and Yuan Dynasties" [7], it is proposed that the regional changes of "Zhang-epidemic" after the song and Yuan Dynasties are due to the expansion of Chinese culture in the local areas and the influence of the Central Plains Dynasty on the local areas. Qiang Ma's "the geography of 'Zhang-epidemic' in the southwest of Tang and Song Dynasties and the understanding and response of the intelligentsia" [8] discusses the intelligentsia's active elimination of the blind fear of "Zhang-epidemic" in the past from the perspective of the geographical environment of the southwest and the south of the five ridges, making full use of medical knowledge to solve the problem and using scientific methods to deal with "Zhang-epidemic". This paper aims to discuss the epidemic situation and treatment measures of "Zhang-epidemic" in Bashu area in Song Dynasty.

\section{The Prevalence of "Zhang-epidemic" in Bashu Area in Song Dynasty}

In the Song Dynasty, social, economic, cultural, scientific and Technological Development flourished. At this time, medicine is also in a period of vigorous development, with a more systematic understanding of "Zhang", "Zhang-gas" and "Zhang-epidemic". For example, "Zhang" and "Zhang-gas" were regarded as the pathogenic factors of "beriberi" and "Zhang-epidemic" in medical works of Song Dynasty. Therefore, there are many names of "Zhang-epidemic", such as the name of "Zhang-epidemic" in Ziming Chen's "Guan Jian Daquan Liangfang" in the Southern Song Dynasty, including smoke "Zhang", haze "Zhang", green grass "Zhang", Huangmao "Zhang", earthworm "Zhang", Wufeng "Zhang", strangled intestine "Zhang", etc. [9]

"Zhang-epidemic" often occurs in the hot, humid, foggy, mountain blocking, sparsely populated and remote areas in the south, which are easy to nourish toxins. Therefore, in ancient South China was a high incidence area of "Zhang-epidemic", especially in Lingnan and Bashu areas. Before the Song Dynasty, "Zhang-epidemic" had been widespread in Bashu area. In the "whole Tang poetry", "Zhang-epidemic" was recorded: "the governor Bafen Yan 'Zhang-epidemic' land, and the clothes of the dynasty caused the smoke of the imperial furnace." In $\mathrm{Fu}$ Du's poems, there are also many descriptions of the "Zhang-epidemic" in Bashu area. In "September 30, the second year of the Dali calendar", "Zhang' floats in three Shu areas, and the wind and cloud darken hundreds of barbarians." Hot three: "the 'Zhang' will not go out, and Lushui will come to the West." It can be seen that "Zhang-epidemic" often exists in Bashu area. By the Song Dynasty, the social economy and culture of the country had entered a peak period of development, and the Bashu area had also been greatly developed. Nevertheless, "Zhang-epidemic" still exists in Sichuan. According to historical data, there are records of "Zhang-epidemic" in Bashu area during the Song Dynasty, especially in the South and west of Sichuan.

In the two Song Dynasties, the records of "Zhang-epidemic" in the Three Gorges area of the Yangtze River decreased to a large extent compared with that in the Tang Dynasty. Only in Daningjian (today's 
Wuxi), Kuimen (today's Fengjie in Chongqing) and Qianzhou, there are records of "Zhang-epidemic". In "Yudi Jisheng", there are only records of "more "Zhang-epidemic" in its land". According to "Yu Di Ji Sheng", there are more "Zhang-epidemic" in Daningjian and Kuimen, you can drink it as tea after cornel boiling water [10]. It's called "spicy tea" to drive away "Zhang-epidemic".

There are records of malaria in Luzhou, Rongzhou (now Yibin), Fushunjian and Liangshan in southern Sichuan. As recorded in "Yu Di Ji Sheng", there is a mount Lufeng in Lujinguan. The mountain is three thousand feet high and full of "Zhang-gas". If you come to the mountain in mid March and April every year, you will surely die. There is no harm in going to this mountain only in early May [11]. Wenyanbo's "WenlugongWenji" records that Luzhou often suffers from "Zhang-epidemic" in March of each year [12]. It can be seen from the historical documents that the "Zhang-epidemic" in Luzhou is very fierce. In the southern part of Sichuan Province, "Zhang-epidemic" is not only frequent in Luzhou, but also in Rongzhou and Fushun.

In the Song Dynasty, "Zhang-epidemic" also happened in the west of Sichuan. Among them, Lizhou, Yazhou, Weizhou and Maozhou are the areas with high incidence of "Zhang-epidemic". In the historical documents, the "Zhang-epidemic" in these four places has been recorded for many times. For example, in the book of "Taiping Huanyu", it is recorded that: in Hanyuan County, Lizhou, the Han River is 120 miles west of Hanyuan County. Every spring and winter, there is "Zhang-epidemic" [13].

\section{Prevention and Control Measures of Song Dynasty Government}

"Zhang-epidemic" epidemic is prevalent in Bashu area, which has a great influence on the local political system, economic development, social stability and health care. In response to the "Zhang-epidemic" epidemic in Bashu area, the Song government took a series of measures to deal with it.

\subsection{On the Economic Side}

Once "Zhang-epidemic" occurred in Bashu area, the Song government would provide economic support first. The government of Song Dynasty would provide economic subsidies for "Zhang-epidemic" in Bashu area. It will also subsidize Bashu area for the treatment of epidemic in other states and governments. In the first month of the fifth year of Yuanfeng (1082), the army stationed in Luzhou died of "Zhang-epidemic", which cost more than one million taxes of the central government of Song Dynasty. In October of the seventh year of Yuanyou (1092), the Song government allocated medicine money to various armies, arranged medical officers to accompany them, prepared medicines according to prescriptions, and distributed them to soldiers [14]. In the thirty second year of Shaoxing (1162), many soldiers suffered from "Zhang-epidemic" due to the garrison of Jiankang, Zhenjiang, Taiping, Jiangzhou and Chizhou. Therefore, in February of the same year, song Gaozong ordered every state to distribute drugs and send doctors to fully treat patients. To prevent "Zhang-epidemic" in Bashu area from money, medicine and so on.

\subsection{Political System}

According to the Song Dynasty medical book "ShengjiZonglu", "Zhang-epidemic" occurs in summer and ends in autumn, or starts in winter and ends in spring. This shows that the occurrence of "Zhang-epidemic" is closely related to the season. The relationship between "Zhang-epidemic" and seasons in Luzhou is mentioned in the"WenlugongWenji". It is also recorded in "SonghuiyaoJigao",that the "Zhang-epidemic" epidemic along the border of Lizhou is very serious [15]. Because of the close relationship between the occurrence of "Zhang-epidemic" and seasons, the Song government adopted the system of "two years and one generation" in order to avoid going to Bashu to take office in the season of concentrated outbreak of "Zhang-epidemic".

\subsection{Military Phase}

"Zhang-epidemic" has a very serious impact on the war. In the second year of Yuanfeng (1079), Luzhou border was invaded by the enemy. The imperial court sent troops to fight. At this time, "Zhang-epidemic" broke out in the army, with the death toll as high as $90 \%$. If a soldier with "Zhang-epidemic" returns home, the whole family will die. In order to prevent and respond to the various epidemics in the garrison, the 
central government of the Song Dynasty sent medical officers to follow the troops, to change every three years, to isolate and treat them, and to dispense drugs to soldiers and civilians [16].

\subsection{Drugs and Medical Books Awarded by the Government of Song Dynasty}

In the aspect of prevention and control of "Zhang-epidemic", it was common that the government of Song Dynasty gave medicine and medical books to prevent and control "Zhang-epidemic". Song Zhenzong in February of the first year (1008) of Dazhong Xiangfu, ordered the medical officers of each state and county in Sichuan Province to prepare the drugs according to the prescriptions in the "Shenghui fang" and then distribute them to the soldiers. In September of the second year of Dazhong Xiangfu (1009), the army and people of Rongzhou and Luzhou suffered from "Zhang-epidemic". The government of Song Dynasty sent officials to reward the people and soldiers of Rongzhou and Luzhou with drugs to ward off "Zhang-epidemic" [17]. Since then, for three years in a row, drugs have been awarded, which shows the importance of "Zhang-epidemic" in Bashu area. The Song government not only gave medicine to the areas that often suffered from "Zhang-epidemic", but also in April of the 8th year of Zhongda Xiangfu (1015) to Rongzhou, Luzhou and Fushunjian, each of them was given a part of "Shenghui fang" to drive away "Zhang-epidemic". In 1051, the Song government ordered all the transportation departments to post the prescriptions in "Shenghui fang" in case of "Zhang-epidemic", so that the people could copy and prevent the "Zhang-epidemic".

\subsection{Study on the Prevention and Control of "Zhang-epidemic" by Bashu Scholars}

During the Song Dynasty, the central government played a leading role in the prevention and control of "Zhang-epidemic" in Bashu area. At the same time, the scholars in Bashu also made indelible contribution to the prevention and control of "Zhang-epidemic". The development of politics, economy, culture and education in the two Song Dynasties provided favorable conditions for the prosperity of medicine in Bashu area. It is in such a background that scholars in Bashu began to study "Zhang-epidemic" in an all-round way. The research of Bashu scholars on "Zhang-epidemic" control can be roughly divided into: first, Bashu scholars who are officials in other places study "Zhang-epidemic". For example, in "Su Shen's good prescription", Su Shi's "Shengsanzi prescription" was widely used in the treatment of "Zhang-epidemic" in the Song Dynasty, and it was included in the "Zhenheshenjizonglu". In the book with Wang Minzhong, there is a record of the treatment of "Zhang-epidemic", which can be treated by boiling ginger, scallion and black bean together. Second, the study of "Zhang-epidemic" in Bashu area by non Bashu officials. For example, Li Fan of the Northern Song Dynasty, who was an official in the south for a long time, wrote the "Theory of 'Zhang-epidemic"'.

\section{Summary}

As a kind of local disease, "Zhang-epidemic" often happened in Bashu area in Song Dynasty, which seriously affected the politics, economy, military and people's daily life. Therefore, in the aspects of financial resources, political system and military epidemic prevention, the Song government carried out special treatment and epidemic prevention for "Zhang-epidemic" in Bashu area. Under the active prevention and control of the Song government, it not only alleviated the "Zhang-epidemic" epidemic in Bashu area, but also provided a favorable environment for the political, economic and military development of the Song government. It also promoted the development of public health in the Song Dynasty, and provided a certain reference for the development of public health in the future.

Acknowledgements. Southwest Minzu University Central University project "study on the response measures of Song government to 'Zhang-epidemic' in Bashu area" (Project No.: 2018yyxs03)

\section{References}

1. Peng Zuo: Discussion on the name and reality of "Zhang" [J], Nankai Journal (PHILOSOPHY AND SOCIAL SCIENCES EDITION), 2011, issue 5.(In Chinese) 
2. Hong Zheng:The change of "Zhang" concept and its influence on the life of ancient Lingnan [J], Guangxi Social Sciences, No. 6, 2017.(In Chinese)

3. Yongmin Cai,Mengting Wang:research and specification of "Zhang" name[J], "Chinese Journal of traditional Chinese medicine (former Chinese Medical Journal)", Vol. 32, No. 10, 2017.(In Chinese)

4. Hanyong Feng:literature study on "Zhang-gas" [J], research trends of Chinese history, No. 5, 2006.(In Chinese)

5. Shengsheng Gong: the preliminary study on the distribution and change of "Zhang-epidemic" in China in 2000[J]. Journal of geography, No. 4, 1993.(In Chinese)

6. Jiawei Fan:population migration in the Six Dynasties and "Zhang-epidemic" in Lingnan area [J]. Sinology research, 1998, No.1.(In Chinese)

7. Peng Zuo's:"Zhang-epidemic" and cultural changes in the song and Yuan Dynasties[J]. Chinese Social Sciences, 2004, No. 1.(In Chinese)

8. Qiang Ma:the geography of "Zhang-epidemic" in the southwest of Tang and Song Dynasties and the understanding and response of the intelligentsia[J]. A review of China's historical geography, 2007, issue 3.(In Chinese)

9. Ziming Chen: a good way to see Daquan, Volume 3, and cure malaria, edited by Weizhong Sheng: Dacheng, chenziming, a famous medical book of Tang, song, Jin and Yuan Dynasties, China traditional Chinese Medicine Press, 2005.(In Chinese)

10. Xiangzhi Wang,Yong Li:Yu Di Ji Sheng, Vol. 57, Sichuan University Press, 2005(In Chinese)

11. Xiangzhi Wang,Yong Li:Yu Di Ji Sheng, Vol. 57, Sichuan University Press, 2005(In Chinese)

12. Tuotuo et al: history of the Song Dynasty, Vol. 7, ZhenzongBenji II, Zhonghua Book Company, 2007, P. 143(In Chinese)

13. Wenchu Wang, et al.: "Taiping Huanyu volume 77", Zhonghua Book Company, 2007(In Chinese)

14. Tao Li: Xu Zizhi Tongjian Changbian, volume 478, Zhonghua Book Company, 2004, P11388.

15. Songji Xu:SonghuiyaoJigao, 53-54 of Fanyi 5, Zhonghua Book Company, 2006, P7793.

16. Tuotuo et al: history of the Song Dynasty, volume 461, biography of Fangji, Zhonghua Book Company, 2007, P13509-13510.(In Chinese)

17. Tuotuo et al: history of the Song Dynasty, Vol. 7, ZhenzongBenji II, Zhonghua Book Company, 2007, P. 135(In Chinese) 